\title{
Criterios de diseño y análisis estructural del Centro de Atención y Gestión de Llamadas de Urgencia 112 Catalunya en Reus
}

\author{
Design criteria and structural analysis of the Center for Attention and \\ Management of Emergency Calls 112 Catalunya in Reus
}

\section{R. Señís ${ }^{*}$}

\section{RESUMEN}

El diseño y análisis estructural del Centro de Llamadas de Urgencia 112 Catalunya constituyó un reto técnico y tecnológico, dadas las características y dimensiones de la estructura resistente, con un claro objetivo: obtener una buena resolución del proyecto sobre la base de que la concepción espacial de un edifico depende también de su concepción resistente. Las necesidades funcionales del edificio dieron paso a una tipología edificatoria singular e innovadora sin pilares en la caja operativa del edificio multifuncional. Para ello, se proyectó una estructura metálica (vigas de celosía) de grandes dimensiones colgadas únicamente de cuatro núcleos de hormigón. El objetivo del presente artículo es exponer los criterios utilizados en el diseño y análisis estructural del proyecto, para atender satisfactoriamente las cuestiones formales y necesidades arquitectónicas, así como funcionales, resistentes y constructivas. El edificio, desarrollado con herramientas BIM, actualmente es un referente en los ámbitos de la Arquitectura e Ingeniería estructural.

Palabras claves: Edificio 112 Reus; relación arquitectura-estructura; herramientas BIM; núcleo de hormigón; estructura metálica; vigas de celosía.

\section{ABSTRACT}

The design and structural analysis of the Center for Attention and Management of Emergency Calls 112 Catalunya was a technical and technological challenge, given the characteristics and dimensions of the resistant structure, with a clear objective: to obtain a good resolution of the project on the basis that the spatial conception of a building also depends on its resistant design. The functional requirements of the building resulted in a unique and innovative architectural typology without columns on the operating box of the multifunctional building. For this, a steel large structure (trusses) hangs only from four concrete cores. The paper's aim is to present the criteria used in the design and structural analysis to successfully address the issues and the architectural, functional, resistant and constructive needs of the project. Currently, the building developed with BIM tools is a reference in the fields of Architecture and Structural engineering.

Keywords: 112 Reus building; architecture-structure relationship; BIM tools; concrete core; steel structure; lattice girders (trusses).

(*) LiTA - Universitat Politècnica de Catalunya (UPC-BarcelonaTech), Barcelona (España).

Persona de contacto/Corresponding author: roger.senis@upc.edu (R. Señís)

Cómo citar este artículo/Citation: Señís, R. (2016). Criterios de diseño y análisis estructural del Centro de Atención y Gestión de Llamadas de Urgencia 112 Catalunya en Reus. Informes de la Construcción, 68(541): e139, doi: http://dx.doi.org/10.3989/ic.15.085.

Licencia / License: Salvo indicación contraria, todos los contenidos de la edición electrónica de Informes de la Construcción se distribuyen bajo una licencia de uso y distribución Creative Commons Reconocimiento no Comercial 3.o. España (cc-by-nc). 


\section{EL EDIFICIO 112 REUS}

El edificio para el Centro de Atención y Gestión de las Llamadas de Urgencia 112 Catalunya ubicado estratégicamente en Reus, junto a la autovía de Tarragona y la autopista AP-7, fue promovido el año 2008 por la Generalitat para centralizar cualquier tipo de emergencia en Cataluña mediante la llamada individual al número 112. De esta forma, se trata de una tipología arquitectónica que reúne todos los organismos encargados de atender y gestionar las emergencias de esta Comunidad de forma más eficaz y coordinada, además de ser el primer equipamiento público del país en obtener una «Certificación LEED» (1). El edificio proyectado por ACXT-IDOM acoge todos los cuerpos implicados en la gestión de las llamadas de urgencia: Operadores 112, Mossos, Bomberos, Protección Civil, Sem y CECAT (Centro de Coordinación Operativa de Cataluña), entre otros. Para ello el proyecto, que cuenta prácticamente con una superficie construida de $15.000 \mathrm{~m}^{2}$, se divide horizontalmente en tres niveles claramente diferenciados (Figura 1a), atendiendo a las necesidades funcionales y de seguridad del conjunto requeridas por la propiedad:

- Zócalo de servicios que alberga la zona de servidumbres e instalaciones principales del edificio, además del aparcamiento, donde este nivel tiene dos plantas adaptándose a la topografía del terreno.

- Espacio público o nivel de acceso.
- Caja operativa superior, con una superficie construida de $6.200 \mathrm{~m}^{2}$, donde se ubican las salas de operadores de los distintos organismos en diferentes niveles (estratos horizontales).

El acceso al edificio se realiza desde el nivel intermedio (espacio público) y desde allí, mediante cuatro núcleos de circulación, se accede a la caja operativa. Cada uno de ellos confecciona uno de los distintos recorridos de comunicación (flujos verticales) del edificio conforme a los grados de privacidad y de seguridad requeridos: visitas externas, trabajadores, mantenimiento y autoridades (1). Las pantallas centrales del núcleo de visitas se elevan por encima del edificio hasta una altura de $46 \mathrm{~m}$ para, además, dar lugar a la torre de telecomunicaciones (Figura 1b). Dichas necesidades proyectuales impulsaron que el edificio se caracterice por su diseño arquitectónico $y$, consecuentemente, por la estructura metálica de gran luz que conforman la caja operativa, ya que ésta gravita únicamente en los cuatro núcleos de circulación que realizan, también, la función resistente del volumen superior (núcleos de hormigón de base rectangular). Las características y dimensiones de la caja operativa $(67,35 \times 36,50 \mathrm{~m}$ en planta y 13,50 m de altura), acorde a las necesidades arquitectónicas expuestas, convergen en una «estructura innovadora que permite prescindir de pilares en el interior del edificio», como expone el arquitecto del proyecto Marco Suárez (2). Este aspecto favorece «la flexibilidad del edificio (plurifuncional) para incorporar futuras distribuciones, a la vez que se potencia una imagen de unidad entre todos los cuerpos operativos» (1).
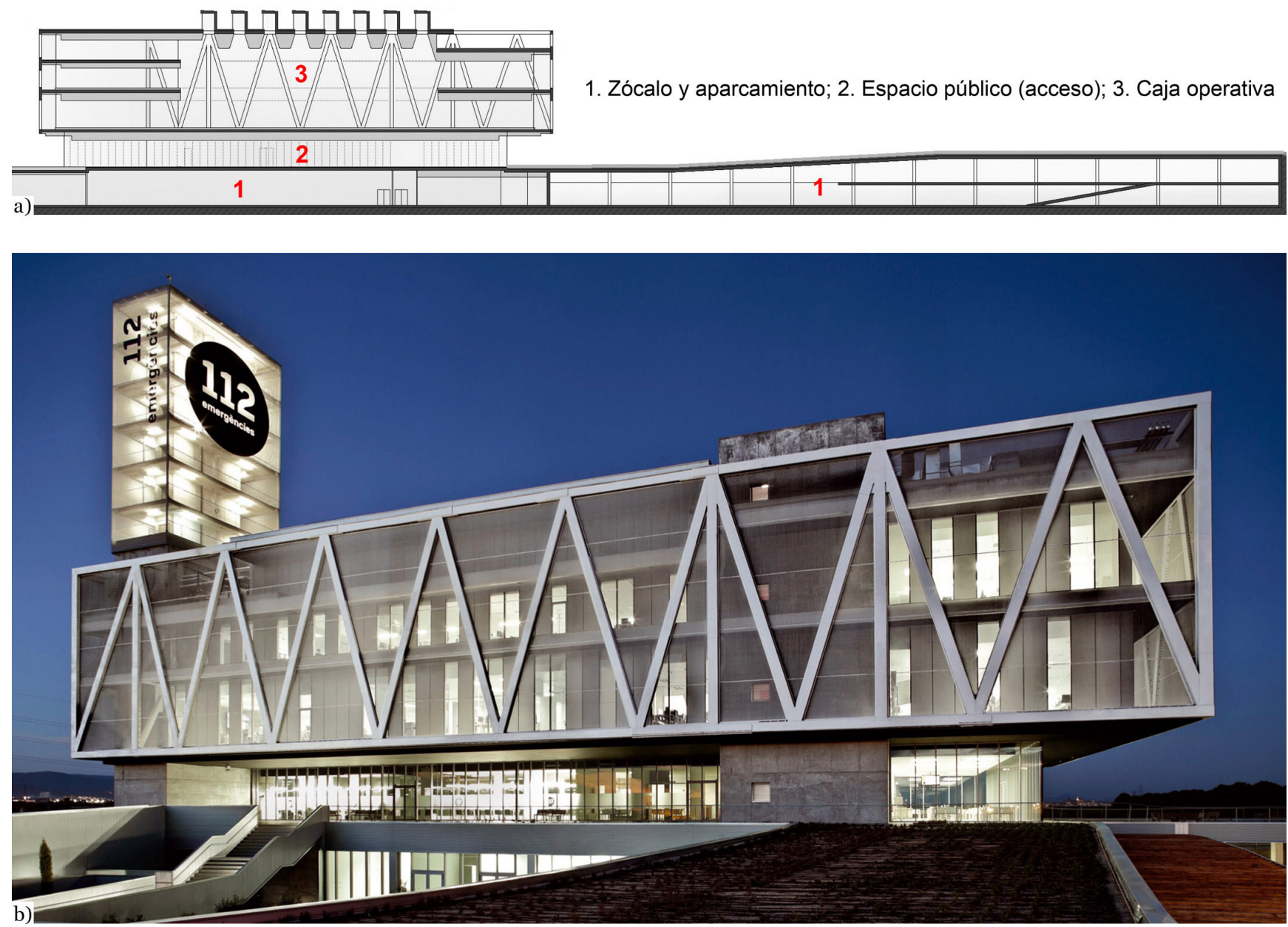

Figura 1. a) Sección longitudinal del conjunto, b) Vista de la caja operativa y torre de telecomunicaciones desde la fachada sur. (Redibujado sobre la base de (1) e imagen cedida por Adrià Goula, respectivamente). 
La tipología estructural proyectada da respuesta a las cuestiones formales y funcionales del edificio 112 Reus (Figura 1b), y se compone de vigas de celosía de grandes dimensiones (luz y canto). Cuatro de ellas dispuestas dos a dos en los costados de los núcleos (vigas longitudinales L1-L4), mientras que las armaduras de cierre se sitúan en los laterales (vigas transversales T1 y T2), de acuerdo a la Figura 2. Las armaduras que se ubican en el perímetro (vigas longitudinales exteriores y vigas transversales) envuelven la caja operativa. Entretanto las dos vigas longitudinales interiores delimitan el patio central o atrio. Espacio alrededor del cual se articulan las distintas salas operativas, aumentando la coordinación y las sinergias entre los distintos operadores (1).

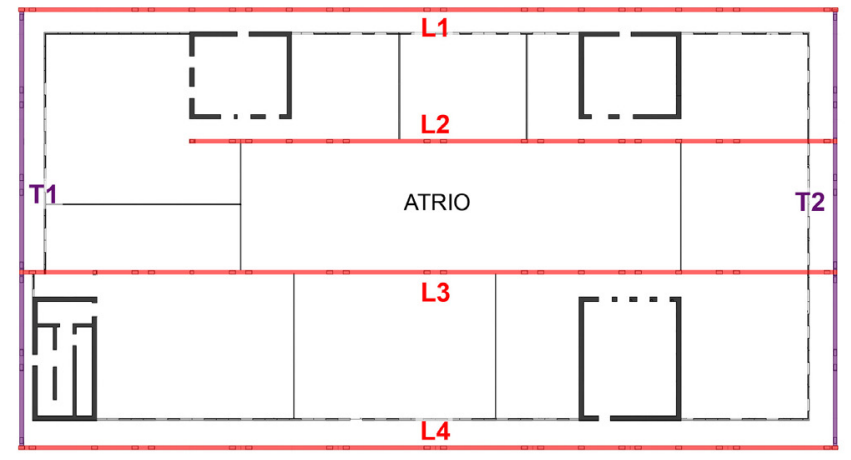

Figura 2. Disposición en planta primera de las armaduras en relación con los núcleos y atrio. La nomenclatura L1-L4 corresponde a las vigas longitudinales. T1 y T2 indican las vigas transversales. (Realización propia).

Consecuentemente, las vigas trianguladas que conforman la estructura resistente de la caja operativa se muestran al exterior (envolvente) e interior (atrio) del edificio. Un ejemplo de cierta relevancia de este concepto arquitectónico es el proyecto del Ayuntamiento de Benidorm, entre otros (3).

En este sentido, el creciente interés en los ámbitos de la Arquitectura e Ingeniería estructural en el uso de sistemas estructurales de acero, como elementos resistentes envolventes -propio de edificios en altura (4) (5)-, comporta que actualmente el concepto estructural adquiera un papel de mayor relevancia en el diseño y estética del edificio. Por consiguiente, para poder hablar de una buena resolución arquitectónica del mismo, es necesaria la existencia de una estrecha relación del binomio que conforman ambas concepciones, diseño arquitectónico y concepto estructural, conocida, ésta, como «estética estructural» (5) (6) (7) (8).

Es por ello que, en acepción al ingeniero de caminos Javier Manterola, "para obtener un buen proyecto, la concepción espacial de un edificio depende también de su concepción resistente» (9) (10).

Este nuevo contexto arquitectónico ha modificado significativamente los parámetros que rigen el papel de la estructura en la concepción del proyecto (11), aspecto de interés que se aborda en mayor profundidad a continuación acorde a la temática presentada, destacando claramente los aspectos relevantes de la relación arquitectura-estructura.

\section{LA RELACIÓN ARQUITECTURA-ESTRUCTURA}

Atendiendo al planteamiento de concepción previamente enunciado, desde un punto de vista formal y compositivo, la característica principal del edificio 112 Reus reside en la dependencia inequívoca entre arquitectura y estructura. Acentuando, así, la necesaria y estrecha colaboración entre arquitecto e ingeniero, para la obtención de un diseño integrador y altamente satisfactorio, a pesar de su complejidad funcional, formal y resistente.

El proyecto se caracteriza por disponer el sistema resistente como envolvente exterior, confeccionando una innovadora estructura contenedora, cuya relevancia reside en los aspectos que se indican a continuación, siendo básicamente:

- Optimización estructural de la composición, en función de la respuesta resistente.

- Utilización de pieles y fachadas resistentes, identificando la estructura como envolvente exterior.

- Liberación del espacio interior (diafanidad).

En definitiva, las estrategias de diseño estructural utilizadas en relación al desarrollo del proyecto dotan al concepto arquitectónico de coherencia, rigor e integridad, mediante la existencia de un único orden compositivo-estructural. De esta forma, se desarrolla el potencial de la fachada portante como sistema configurador del edificio (11).

Esta característica se potencia al disponer en la estructura perimetral una malla textil Serge Ferrari, atendiendo a cuestiones arquitectónicas, que genera la piel envolvente aprovechando las posibilidades que ofrece el concepto estructural. Dicha solución, no sólo aporta una plusvalía estética sino que además responde a las exigencias en materia de protección térmica, acústica y luminosa, mejorando el confort y la privacidad de los usuarios. Resolviendo, además, la problemática de compatibilidad clásica acerca de la colocación del cerramiento respecto al sistema resistente, al disponerlo en un plano ulterior (doble piel), generando, a su vez, un recorrido perimetral de servidumbre y mantenimiento de la estructura y/o fachadas (Figura 2). Además, este espacio permite albergar los elementos transversales de sujeción de las armaduras (apartado 3.3), dotando de protagonismo a la estructura al utilizar su respuesta resistente como medio definidor de la concepción espacial (12).

En este contexto, destacar que la disposición de las vigas de celosía, de acuerdo a lo anteriormente expuesto, configuran espacios interiores diáfanos al separar las armaduras L1-L4 entre 10,80 y 14,40 m, según el caso, permitiendo una gran flexibilidad plurifuncional en cuanto a la distribución de las distintas salas y estancias de la caja operativa y nivel intermedio, atendiendo a las cuestiones arquitectónicas y funcionales de este singular e innovador edificio. Asimismo, el sistema estructural posibilita colocar, indistintamente en altura, los forjados de la caja operativa a distintos niveles (apartado 3.4), comunicados verticalmente por los propios núcleos resistentes. También permite generar espacios libres (sin forjados intermedios) de doble y triple altura para la correcta supervisión de la salas CECAT y operativa, entre otras. Además, estas zonas estructuralmente aligeradas se colocan en puntos estratégicos, descargando los elementos del sistema resistente más comprometidos (voladizos) y solicitados. De igual forma, el atrio responde a dicha sensibilidad proyectual, a la vez que actúa como 
punto central de entrada de luz natural (lucernarios). Estas estrategias son algunos ejemplos de diseño arquitectónico-estructural que generan una indudable interacción bidireccional integral en todo momento entre ambas vertientes.

En efecto, la estructura adquiere, además de su función estática y resistente, un destacado papel en el diseño y la composición arquitectónica del proyecto, influyendo significativamente el concepto estructural en la concepción del mismo. Definiendo, pues, la forma de éste a través de geometrías que optimizan el comportamiento resistente, atendiendo a criterios de eficiencia estructural, se establece un sistema único e innovador, ad hoc, para un fin determinado. No siendo el objetivo resolver óptimamente, desde la forma/geometría, un problema general como es propio de construcciones tradicionales (arcos, bóvedas, cúpulas, etc.) (11).

Siendo la composición y sistema resistente del edificio 112 Reus resultado de la eficiencia estructural (aunque no necesariamente estricta u óptima), basándose en la naturaleza de los esfuerzos para definir y configurar el proyecto, reconociendo formalmente, el flujo racional de las cargas, como se expone en el apartado 3.3, en relación al diseño de las vigas de celosía.

A su vez, el planteamiento formal y de optimización utilizado permite abrir innovadoras líneas de investigación para afrontar nuevos retos arquitectónicos mediante un mayor número de posibilidades proyectuales. Frecuentemente, con el soporte informático de sistemas BIM (Building Information Modeling), dada la complejidad en el diseño y construcción que conlleva este tipo de configuraciones, sobre la base de novedosas geometrías propias de los edificios de nueva generación. Por ello, se deriva la necesidad de utilizar herramientas paramétricas de modelado compatibles con la tecnología y programas avanzados de $\mathrm{CAD}$, así como de análisis estructural. Estas nuevas y/o evolucionadas herramientas BIM, utilizadas en el desarrollo del proyecto (planificación, diseño, construcción y gestión), han permito cambiar los procesos de producción y métodos de entrega en la industria de la Arquitectura, Ingeniería y Construcción (8), con una innovadora plataforma de trabajo.

En el caso concreto del edificio 112 Reus, el uso de este tipo de herramientas de modelado permitió la correcta y necesaria coexistencia formal entre arquitectura y estructura, implementando inequívocamente aspectos esenciales de colaboración, como los que se indican a continuación, entre arquitectos e ingenieros:

- Mayor eficiencia del equipo de trabajo.

- Flexibilidad de trabajo mediante el intercambio de información (exportación de modelos).

- Posibilidad de integración con funciones API (Application Programming Interface), para asegurar un mayor nivel de compatibilidad.

Para ello, se utilizó el Revit Architecture como herramienta de trabajo conjunta, con un único modelo para introducir los distintos sistemas configuradores del proyecto (arquitectura, estructura, instalaciones, etc.). Posteriormente, mediante el módulo Revit Structures se definieron con mayor detalle las características geométricas y mecánicas (material, dimensiones, etc.) de los distintos elementos resistentes, previo a la extensión de dicha plataforma para la interacción con productos de Ingeniería estructural CSI (Computer \& Structures, Inc.). Exportando con plugins el modelo realizado a progra- mas de análisis como SAP2000, SAFE y TEKLA, cuya aplicación se expone en el apartado 4.1, según el elemento resistente a analizar y dimensionar. Una vez realizado el análisis final del sistema estructural completo, se importó la información obtenida (dimensionado) para actualizar el modelo inicial (Revit Architecture), detectando, así, los problemas e interferencias entre los distintos sistemas del proyecto, obteniendo una completa integración arquitectónico-estructural.

En definitiva, fue posible el intercambio de datos entre modelos BIM/CSI de forma eficaz, aportando un valor añadido al proyecto, configurando en todo momento un necesario diseño integrador, dada la complejidad tecno-tecnológica y de gestión del mismo.

En este sentido, en el momento de afrontar el desarrollo y la ejecución de un proyecto de estas características es oportuno definir de forma clara los criterios de diseño y análisis estructural, los cuales se exponen en los siguientes apartados.

En efecto, el objetivo del presente artículo es exponer los criterios de mayor relevancia utilizados en el diseño y análisis estructural, del proyecto expuesto, para atender satisfactoriamente las cuestiones formales y necesidades arquitectónicas, así como funcionales, resistentes y constructivas del mismo. Por ello, también se destacan los aspectos significativos derivados del proceso de ejecución.

\section{CRITERIOS DE DISEÑO DE LA ESTRUCTURA RESISTENTE}

A tenor de lo previamente enunciado, en el presente apartado se describen los criterios de diseño de la estructura. Conforme a las características del edificio, anteriormente expuestas, su matriz funcional (estratos horizontales y flujos verticales) concurre en la concepción resistente del mismo. En este contexto es oportuno indicar los sistemas estructurales principales del proyecto:

- Forjado reticular de hormigón armado para los techos de la planta zócalo.

- Estructura metálica con núcleos de hormigón y forjados prefabricados (placas alveolares) para la caja operativa.

Debido a la singularidad de la estructura proyectada, consecuentemente, como se expone en el siguiente apartado 3.1, para cada sistema resistente se proyectó un tipo de cimentación específico, claramente diferenciadas entre ellas, adaptándose a las características del edificio en cada punto. Las distintas tipologías estructurales que conforman el edificio se describen técnicamente a continuación, destacando los puntos de mayor relevancia.

\subsection{La cimentación del conjunto}

Acorde a lo previamente expuesto, de igual forma que en la estructura del edificio, en este punto es necesario diferenciar las respectivas cimentaciones del zócalo y núcleos.

La primera consiste en una cimentación superficial de zapatas de base cuadrada que reciben las cargas de la estructura reticular del zócalo. Las zapatas se arriostran, no solo, para resistir los momentos flectores en la base de los pilares de hormigón del zócalo sino, también, los esfuerzos y momentos propios de la acciones sísmicas a considerar en edificios 
de importancia especial, siendo el caso. El hormigón definido para esta cimentación fue HA-25/P/20/IIa.

El segundo tipo corresponde a la cimentación profunda que transmite adecuadamente al suelo las elevadas cargas concentradas en los núcleos resistentes. Ésta se realizó con pantallas de hormigón armado de $60 \mathrm{~cm}$ de grosor encastadas en el terreno, en todo el perímetro de los núcleos. Según las cargas resistidas por cada uno de ellos, la profundidad de las pantallas oscila entre 10-14 m, trabajando por fuste y por punta, de acuerdo a las especificaciones del geotécnico. Para las pantallas se utilizó HA-30/F/12/IIa, al igual que los núcleos, facilitando así su puesta en obra con bomba, justo después de la excavación con bivalva.

\subsection{Forjado reticular (zócalo)}

Las características y usos del zócalo permitieron proyectar una retícula de pilares de $8 \times 8 \mathrm{~m}$ prácticamente en toda la planta. Esto posibilitó realizar una estructura convencional de forjados reticulares de $30+5 \mathrm{~cm}$ de canto y $85 \mathrm{~cm}$ de intereje (nervios), atendiendo a las cargas correspondientes de aparcamiento en la planta intermedia y de cubierta ajardinada en la superior. Los casetones de hormigón y recubrimientos nominales de los nervios garantizan la estabilidad y resistencia de la estructura frente al fuego en este punto (RF-120).

Dadas las dimensiones totales del zócalo en planta $(170 \times 32 \mathrm{~m})$, se dispusieron juntas de dilatación transversales para obtener tramos continuos inferiores a los $40 \mathrm{~m}$ de longitud para, así, no considerar en el análisis de dicha estructura los efectos térmicos, de acuerdo a la normativa consultada.

\subsection{Estructura metálica y núcleos de hormigón (caja operativa)}

En base al planteamiento expuesto, para que la concepción resistente diera respuesta al concepto arquitectónico y necesidades funcionales del edifico, se proyectó una estructura metálica de vigas de celosía de grandes dimensiones, las cuales se apoyan en los cuatro núcleos de circulación/resistentes de $40 \mathrm{~cm}$ de grosor (núcleos de hormigón). Es oportuno constatar que la posición estratégica de los núcleos, desde un punto de vista funcional del edifico y resistente de la estructura, dotan a la caja operativa de grandes voladizos disminuyendo, así, la distancia entre soportes de las vigas trianguladas longitudinales. Esta estrategia de diseño mejora considerablemente su comportamiento estructural, tanto a deformaciones (flechas) como a nivel tensional de las armaduras y las barras que las conforman.

En este contexto, es oportuno destacar la dicotomía surgida en el momento de proyectar la intersección entre las vigas de celosía de acero y los núcleos resistentes de hormigón. Era necesario, pues, establecer que elemento (viga o núcleo) era el de mayor jerarquía y, consecuentemente, debía tener continuidad estructural con respecto al otro. Dada la relevancia de ambos sistemas, así como los materiales definidos para cada uno, una vez valoradas las diferentes opciones constructivas, se optó por proyectarlos en planos distintos, sin interrupciones constructivas. De esta forma, las vigas trianguladas cuelgan de los costados perpendiculares de los núcleos, respecto el plano principal de éstas, mediante los elementos estruc- turales denominados «orejeras transversales» (Figura 3a). Asimismo, este aspecto permitió ejecutar los núcleos de hormigón de forma continua en toda su altura con encofrados autotrepantes (Figura 3b).
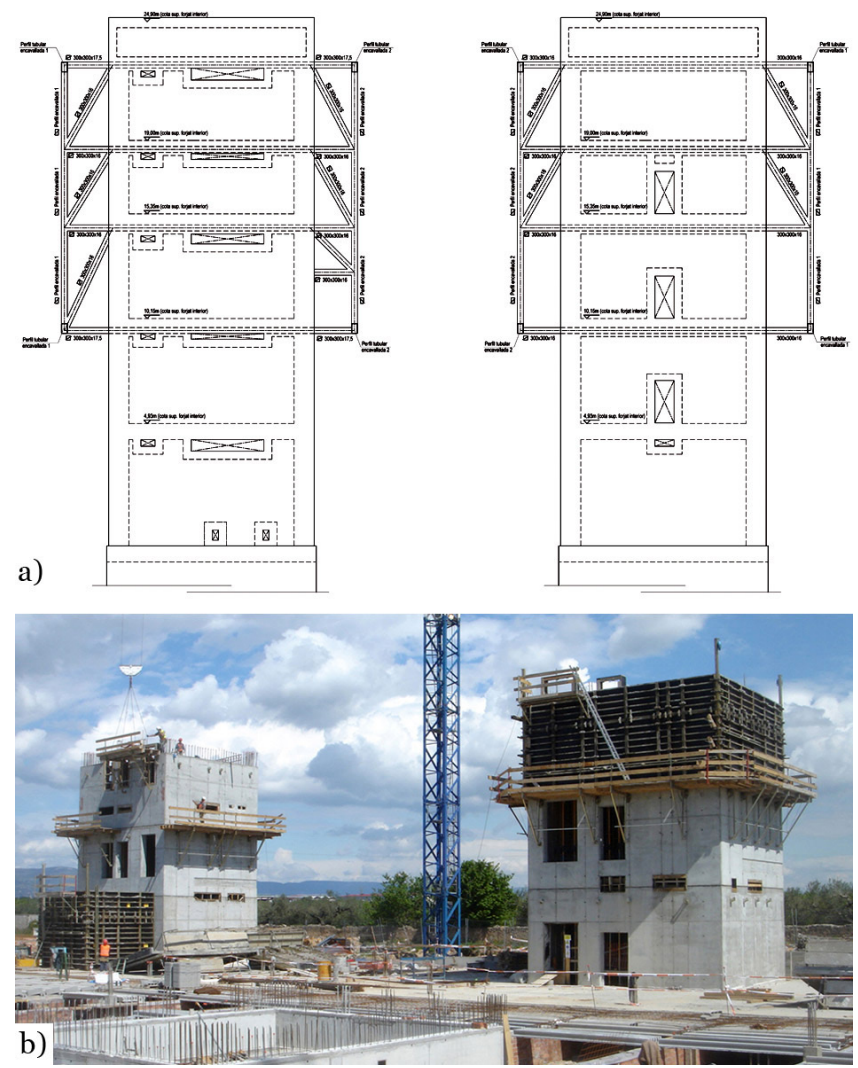

Figura 3. a) Geometría de los alzados este y oeste del núcleo de visitas con las «orejeras transversales» de sujeción de las armaduras

longitudinales, b) Ejecución de los núcleos con encofrado autotrepante. (Realización e imagen propias).

Las «orejeras transversales» mostradas en la Figura 3a corresponden al núcleo de visitas. Estos elementos de sujeción de las armaduras longitudinales se proyectaron también con vigas trianguladas de acero. Menos en aquellos puntos donde se eliminaron las diagonales que impedían la correcta circulación de las personas.

En cuanto a las vigas de celosía se refiere es oportuno mencionar las características geométricas principales de éstas, representadas en la Figura 4a, y que se indican a continuación:

- Vigas trianguladas de $13,50 \mathrm{~m}$ de canto (altura total caja operativa).

- Vigas de celosía tipo Warren con montantes intercalados, únicamente, donde se unen con las «orejeras transversales» (núcleos).

- Distribución en alzado de los forjados de la caja operativa (estratos horizontales) en cinco niveles distintos. Dos de ellos ubicados en los cordones de las vigas y el resto dispuestos en puntos intermedios de las armaduras, según las necesidades arquitectónicas.

Las características geométricas y prestaciones mecánicas de las vigas de celosía proyectadas dieron respuesta al concepto arquitectónico (concepción espacial) y a los requerimientos estructurales (concepción resistente), incidiendo en el razonamiento expuesto de Javier Manterola (9) (10). 

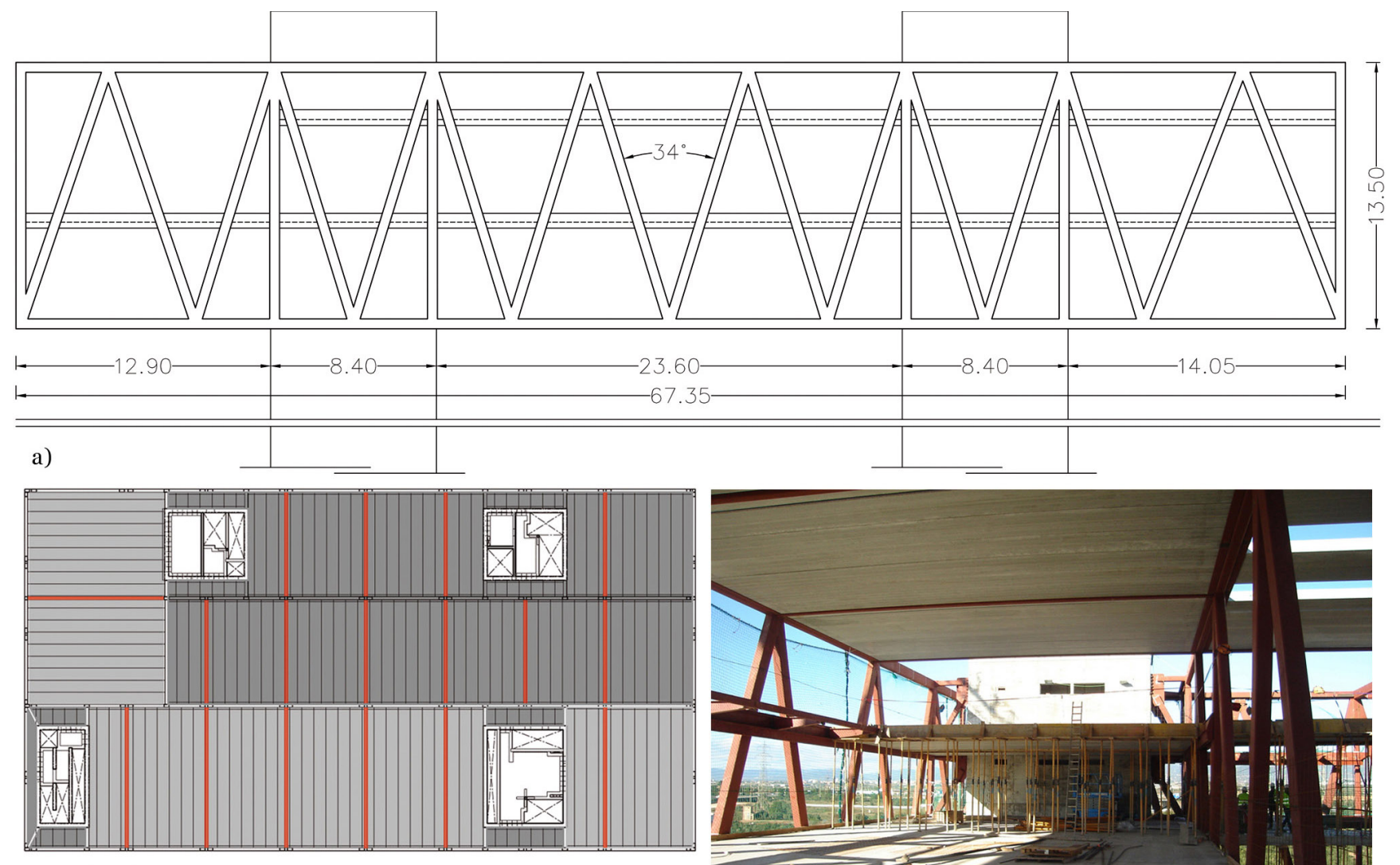

b)

Placa Alveolar $25+5$

Placa Alveolar $35+5$

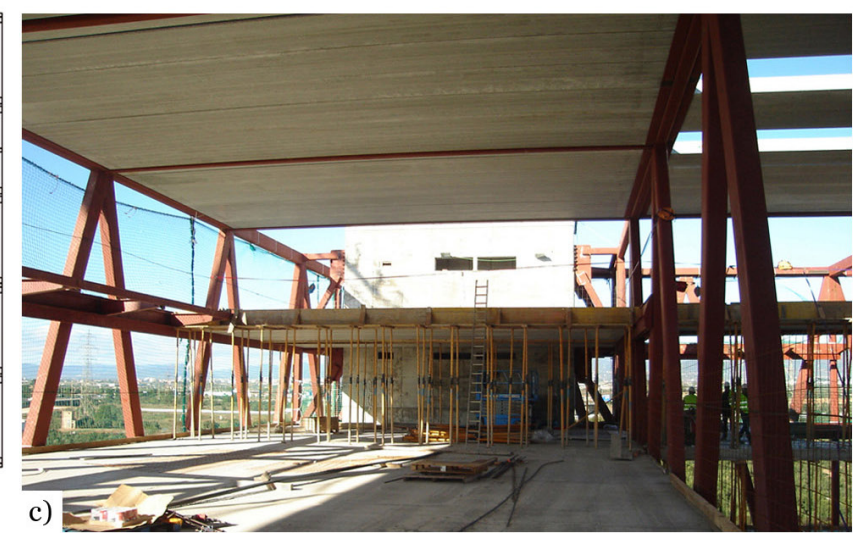

Figura 4. a) Geometría exterior de la viga Warren correspondiente a la celosía L1 (Figura 2), b) Distribución de las placas alveolares de distintos cantos en el primer nivel de la caja operativa (en color rojo se indican los elementos de arriostramiento entre los cordones inferiores de las armaduras), c) Placas alveolares colocadas. (Realizaciones e imagen propias).

Desde el punto de vista estético, las vigas Warren se consideran de aspecto agradable en los ámbitos de la Arquitectura e Ingeniería estructural debido a su ligereza visual, ya que su malla menos tupida hace que su diseño sea muy apreciado por arquitectos e ingenieros, en comparación con otras geometrías de armaduras. Mientras que, atendiendo al aspecto resistente, con esta tipología se alcanzó optimizar las vigas trianguladas desde la ejecución (8) (13) (14) (15) (16), así como desde la eficiencia estructural, sobre la base de la influencia de la geometría o disposición de las barras de relleno si éstas identifican, en la medida de lo posible, los esfuerzos axiales propios de la transmisión racional de las cargas a soportar hasta los apoyos (8) (17).

Por estas razones, en cuanto a las prestaciones y comportamiento estructural de la viga Warren se refiere, esta armadura resulta relativamente ligera y altamente eficiente presentando, así, una elevada resistencia mecánica respecto al coste de los materiales utilizados en su construcción (8) (13) (14) (17).

En este contexto, es de interés el análisis de la influencia de la geometría en la rigidez y eficiencia estructural de vigas de celosía de acuerdo a los distintos parámetros de diseño, identificando las direcciones de los esfuerzos principales que las solicitan (8) (17) (18) (19) (20) (21). Temática abordada ampliamente en el ámbito científico-técnico, especialmente desde la optimización del tamaño, la forma o la topología (22) (23) (24) (25), entre otros.

Conforme al planteamiento de optimización expuesto, en la Figura 4a se muestra la geometría de la viga Warren de la celosía
L1 (Figura 2), como exponente significativo de esta concepción. Ésta se compone de triángulos isósceles con ángulos entre diagonales próximos a los $30^{\circ}$. Esta disposición de las diagonales mejoró de forma considerable el comportamiento estructural de las vigas trianguladas. El cual se obtuvo al reducir la longitud de pandeo de los cordones comprimidos y la distancia entre apoyos de las vigas continuas que reciben los forjados intermedios (apartado 3.4), sin la necesidad de colocar montantes verticales en todos los recuadros. Aspecto que habría dificultado y encarecido excesivamente la ejecución (8) (13).

En este sentido, mencionar que se escogieron perfiles tubulares huecos de sección cuadrada/rectangular para confeccionar las vigas de celosía, ya que éstos son altamente eficaces a esfuerzos axiales y cortantes, así como a los momentos flectores y torsores considerados en el análisis de la estructura metálica. Éstos últimos debido a la excentricidad producida entre el punto de apoyo de los forjados (placas alveolares) con respecto al c.d.g. de la sección resistente de los perfiles tubulares de las distintas barras de las armaduras.

En cuanto a los perfiles tubulares, referidos previamente, indicar que se utilizaron secciones de $500 \times 300 \mathrm{~mm}$ con espesores de entre 8-25 mm para las barras de las armaduras. Dichas dimensiones implicaron, en algunos casos, considerar en el análisis las características mecánicas y resistentes de las secciones eficaces de los perfiles de clase 4 , de acuerdo a la normativa consultada. En el caso de las «orejeras transversales», se emplearon perfiles de $300 \times 300 \times 16 / 18 \mathrm{~mm}$. En todo caso, escogiendo aquellos de menor peso que cumplieran con las condiciones de dimensionado correcto para Esta- 
dos Límite Últimos, ELU, y que dieran una respuesta satisfactoria al cumplimiento de la normativa para Estados Límite de Servicio, ELS. Para todos ellos, el acero definido fue S-355.

En este punto destacar, por su relevancia en la estabilidad de las vigas trianguladas, los elementos de arriostramiento (perfiles perpendiculares al plano de las armaduras) colocados, principalmente, entre los cordones de las vigas longitudinales (Figuras 4bc), frente al pandeo global e inestabilidad lateral de éstas. Fenómenos propios de estructuras de gran luz (esbeltez horizontal L/H considerable) al entrar en carga, especialmente en el caso estudiado, durante su montaje y posterior colocación de los forjados prefabricados.

\subsection{Los forjados de la caja operativa}

Conforme a lo expuesto anteriormente, la caja operativa se distribuye en cinco niveles a distintas alturas o posiciones con respecto las armaduras. Dichos niveles se confeccionaron con forjados de placas alveolares (Figuras 4bc) que se apoyan lateralmente en las vigas de celosía, mediante soportes continuos. Esta tipología constructiva se planteó para facilitar el montaje en obra y reducir los plazos de ejecución, al tratarse de un sistema estructural prefabricado autoportante.

Las dimensiones de las placas alveolares utilizadas fueron $120 \mathrm{~cm}$ de ancho y 25-40 cm de canto, más su correspondiente capa de compresión ( $5 \mathrm{~cm}$ de hormigón armado). Las características geométricas descritas atienden, en cada caso, a la luz a cubrir (separación entre armaduras) y las cargas a soportar, según la zona.

En cuanto a los soportes continuos que sustentan las placas alveolares, se utilizaron perfiles en «L» (Figura 5a) cuando éstos coincidían con los perfiles tubulares de los cordones. Mientras que, para los forjados apoyados en puntos intermedios de las armaduras (diagonales/montantes) se diseñaron soportes continuos específicos: perfiles en «E», dada su geometría en sección (Figura 5b). Cuyo análisis, mediante el método de elementos finitos (MEF), se expone en el apartado 4.2. Para ambos elementos se definió acero S-275.

En la Figura 5b se muestran las secciones transversales de los tres soportes tipo de los niveles intermedios de la caja operativa, y por consecuente, que se apoyan en las diagonales/ montantes de las armaduras. Las denominaciones E-850, E-80o y E-750 especifican la altura total del elemento resistente acorde a su dimensionado correcto (ELU) según las cargas a soportar y la luz a cubrir, en cada punto.

De igual forma que los perfiles tubulares huecos que conforman las vigas trianguladas, en los soportes en «E» se optó por diseñar un cajón cerrado en la parte inferior de la pieza, para resistir los momentos torsores que solicitan este elemento estructural debido a la carga excéntrica de las placas alveolares, además de los momentos flectores propios de una viga continua. Indicar que la platabanda superior de los soportes se colocó frente al pandeo local de la pletina vertical en los tramos de momentos flectores positivos (cabeza superior comprimida).

Conforme a los distintos elementos descritos de la estructura metálica proyectada, el peso propio total del acero estructural es de $600.000 \mathrm{~kg}$, resultando un ratio de $96,80 \mathrm{~kg} / \mathrm{m}^{2}$ construido (caja operativa), para unas sobrecargas de uso iguales

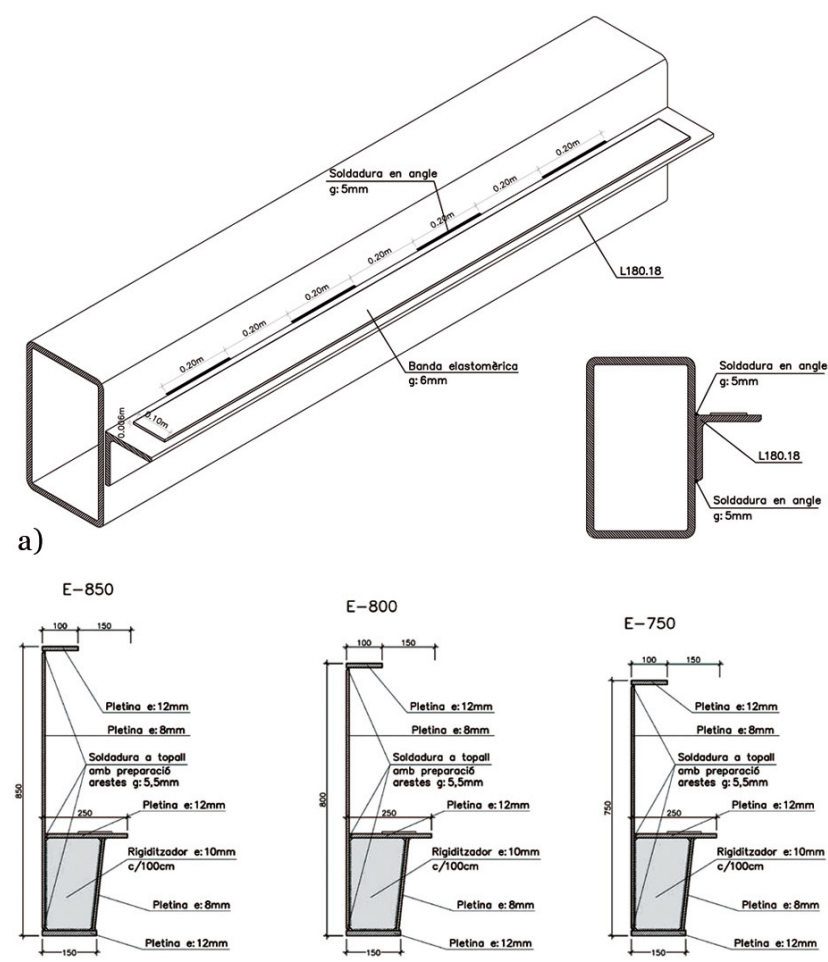

b)

Figura 5. Secciones resistentes de los soportes para los forjados de la caja operativa: a) Soportes en «L» coincidentes con los cordones, b) Soportes en «E» para los niveles intermedios. (Realización propia).

o superiores a 3,00 $\mathrm{kN}$ en todo el edificio, estableciendo unas cargas totales entre 9,70-14,00 kN/m², según la zona.

En cuanto a la construcción de la estructura metálica, subrayar que se desarrolló esencialmente en obra, a petición de la constructora, aspecto que requirió un esfuerzo tecno-tecnológico y humano notables, así como económico (control de calidad y seguridad), para garantizar su correcta ejecución, siendo necesarios elementos de apuntalamiento y arriostramiento especialmente diseñados. Asimismo, la existencia del techo planta zócalo, una vez se inició el proceso de montaje de dicha estructura, supuso apuntalarlo considerablemente para soportar las cargas de la maquinaria pesada necesaria en el montaje de los distintos elementos de acero estructural.

Destacar que todas las uniones de la estructura metálica se proyectaron con soldaduras a tope, en el marco de la estética de la estructura y, consecuentemente, del edificio. En este sentido, se abrieron ventanas de trabajo en los perfiles tubulares que requerían pletinas de refuerzo según los esfuerzos solicitantes, para que éstas se colocaran en el interior. En este sentido, indicar que la protección frente al fuego se realizó con pinturas intumescentes.

Mencionar que los criterios del sistema resistente, descritos previamente, se establecieron de acuerdo a los requerimientos arquitectónicos, de ejecución y específicos de las normas básicas consultadas: Código Técnico de la Edificación, CTE (26), y Norma de Construcción Sismorresistente, NCSE (27).

\section{ANÁLISIS ESTRUCTURAL Y RESULTADOS}

A continuación, se exponen los criterios de mayor relevancia considerados, así como los correspondientes resultados, en 
el análisis estructural del edificio 112 Reus. Para ello, se realizaron modelos globales y específicos que se describen en los apartados 4.1 y 4.2 .

\subsection{Análisis estructural}

En el caso del modelo global o completo de la caja operativa se efectuó un análisis dinámico mediante el método modal espectral (o método de la respuesta espectral) utilizando el programa SAP200o, determinando los espectros «envolventes» (28) (29).

Asimismo, acorde a lo indicado en el apartado 2, la utilización del SAP200o permitió intercambiar información con otros programas BIM para una mayor definición, análisis especifico y dimensionado de los diferentes elementos resistentes: ETABS para la estructura reticular del zócalo, SAFE para la cimentación y núcleos de hormigón y TEKLA para detallar la estructura metálica, así como sus uniones, antes de su montaje. Posteriormente, se traspasó la información de los modelos de análisis (dimensionado) al modelo arquitectónico inicial (Revit Architecture), de acuerdo a la metodología BIM aplicada y expuesta anteriormente (apartado 2).

En este sentido, es conveniente destacar que la utilización de dichas herramientas BIM no sustituyó el criterio del responsable del diseño y análisis del sistema resistente, ya que se requirió un alto conocimiento estructural para abordar el correcto dimensionado del mismo. Siendo necesario definir adecuadamente las características geométricas y mecánicas de los distintos elementos estructurales, así como las condiciones de contorno y dimensionado oportunas, entre otros aspectos de relevancia.

\subsection{Modelos estructurales}

En el presente apartado, se exponen los modelos más significativos realizados con el SAP200o para el análisis de la estructura del edificio 112 Reus. El modelo completo de la caja operativa (Figura 6) se compuso de 3.109 barras que modelizaban, principalmente, los elementos metálicos y 22.632 placas correspondientes a los elementos de hormigón armado (núcleos y forjados).

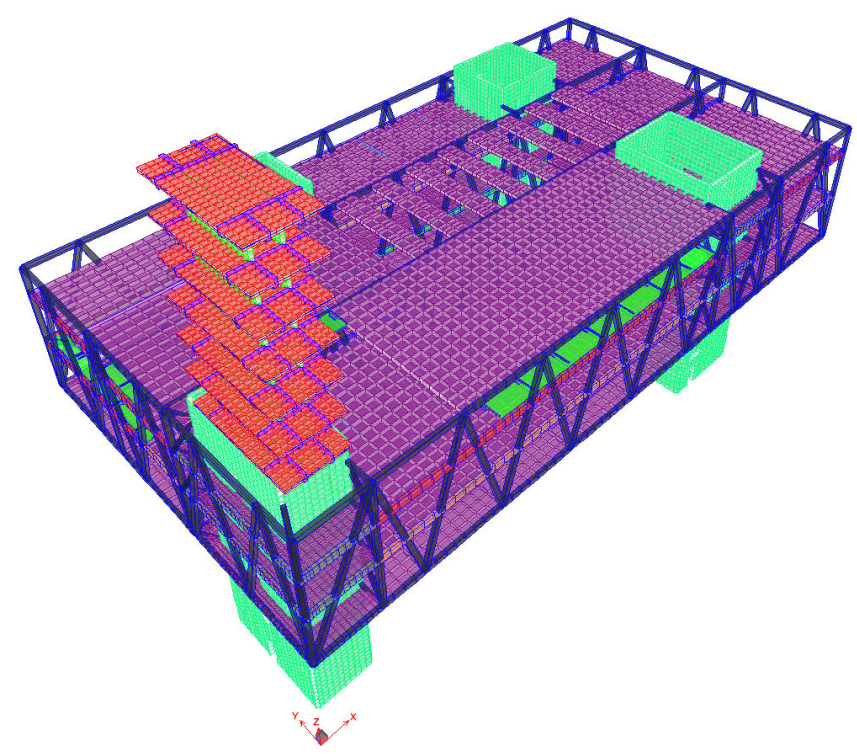

Figura 6. Vista 3D del modelo completo de la caja operativa. (Imagen propia tomada del SAP2000).
Generalmente, las uniones entre las distintas barras del modelo se definieron como rígidas. Únicamente la unión entre los núcleos resistentes y la estructura metálica se modelaron mediante rótulas (liberando los momentos en los tres ejes), y cuya ejecución se realizó con pletinas verticales que permitían dicho comportamiento estructural.

En cuanto a los núcleos de hormigón, se analizaron los flujos axiales y las tensiones principales, para determinar el armado necesario en cada punto. De igual forma, se utilizó el referido SAFE para obtener valores globales de estos elementos para analizar su comportamiento estructural y dimensionar la correspondiente cimentación.

Dada la singularidad geométrica de algunos elementos resistentes de la estructura, éstos se analizaron con modelos específicos mediante el MEF, en régimen lineal. Es el caso de los soportes en «E» de las placas alveolares sobre las diagonales/ montantes de las armaduras. Previo a su análisis se generaron, con herramientas $\mathrm{CAD}$, las geometrías malladas (discretización) compuestas por elementos finitos. Posteriormente, se definieron en el SAP200o sus características geométricas y mecánicas, previo al análisis estático y dimensionado, según las cargas correspondientes. Asimismo, se analizaron las uniones más solicitadas, para reforzar con pletinas interiores aquéllas en las que fuera necesario.

Destacar que, el caso los soportes en «E», se introdujeron los movimientos propios de las vigas trianguladas en los puntos de unión con estos elementos, aplicando los desplazamientos y giros correspondientes en los tres ejes. Para así, también, considerar los esfuerzos resultantes de dichos movimientos mediante el método de la rigidez.

Los resultados representativos de los distintos modelos expuestos y detalle de ejecución se muestran en el apartado 4.3 .

\subsection{Resultados representativos}

A continuación, se presentan los resultados representativos de los distintos modelos de análisis realizados. En la Figura
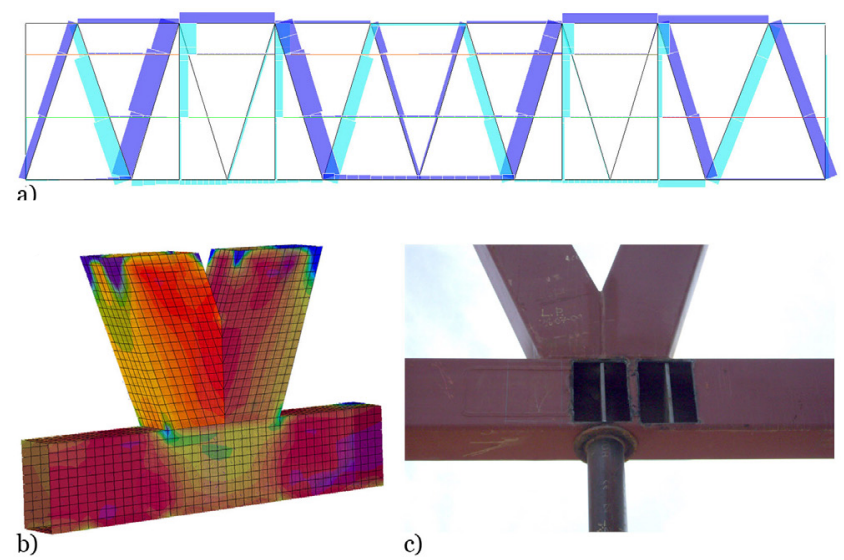

Figura 7. Resultados representativos obtenidos: a) Esfuerzos axiales (ELUo12) de la celosía L1 (Figura 2). En azul oscuro se representan los esfuerzos de tracción y en azul claro los esfuerzos de compresión. b) Diagrama de tensiones «Von Mises» de unión en «V» mediante el MEF, c) Ejecución de unión en «V» y sistema apuntalado. (Imágenes propias tomadas del SAP200o y en obra, respectivamente). 
7a se muestra el diagrama de esfuerzos axiales de la armadura L1 (Figura 2), para la combinación ELUo12. En la cual, la sobrecarga de uso fue la acción principal y en las sobrecargas restantes se aplicaron sus respectivos coeficientes de simultaneidad, según (26).

El resultado del MEF (ELUo12), mostrado en las Figura 7b, corresponde al diagrama de tensiones «Von Mises» de una unión en «V» en el cordón inferior, así como su correspondiente ejecución, en este caso, con pletinas de refuerzo interiores sobre sistema apuntalado (Figura 7c).

\section{CONCLUSIONES}

Para concluir, se expresan las reflexiones surgidas del trabajo desarrollado, y presentado, para el diseño y análisis del edificio 112 Reus, así como de su posterior ejecución:

a) Constatar, acorde al resultado final obtenido, que la estructura proyectada responde satisfactoriamente a las cuestiones formales y necesidades arquitectónicas, así como funcionales, resistentes y constructivas del proyecto.

b) Subrayar que el edificio ofrece, desde las estrategias de diseño estructural utilizadas, una mayor integridad conceptual, dotando de coherencia y rigor la forma compositiva, potenciando la relación arquitectura-estructura.

c) Mencionar que si bien la estrecha relación profesional entre arquitecto-ingeniero es necesaria en el desarrollo de cualquier proyecto, cuando la propuesta arquitectónica sea menos frecuente es fundamental y esencial intensificar dicha colaboración (10), para garantizar un resultado satisfactorio en todos los aspectos.

En este sentido, la utilización de herramientas paramétricas y programas BIM facilitó la mencionada relación entre profesionales que, a la vez, permitió hacer más eficiente el desarrollo del proyecto. Disminuyendo, así, la pérdida de tiempo en el diseño y la construcción del mismo (8) (30).

d) Considerar admisible el ratio obtenido, de acero estructural, inferior a los $100 \mathrm{~kg} / \mathrm{m}^{2}$ construido (caja operativa), a tenor de las características y dimensiones del proyecto $\mathrm{y}$, consecuentemente, del sistema estructural, para unas cargas totales a soportar de entre 9,70-14,00 kN/m², según la zona.

e) Enfatizar que el edificio proyectado constituyó un reto técnico y tecnológico de gran magnitud, tanto en fase de proyecto como durante el proceso de ejecución (Figura 8a). Actualmente, el edificio 112 Reus es un referente en los ámbitos de la Arquitectura e Ingeniería estructural (Figura $8 b)$.
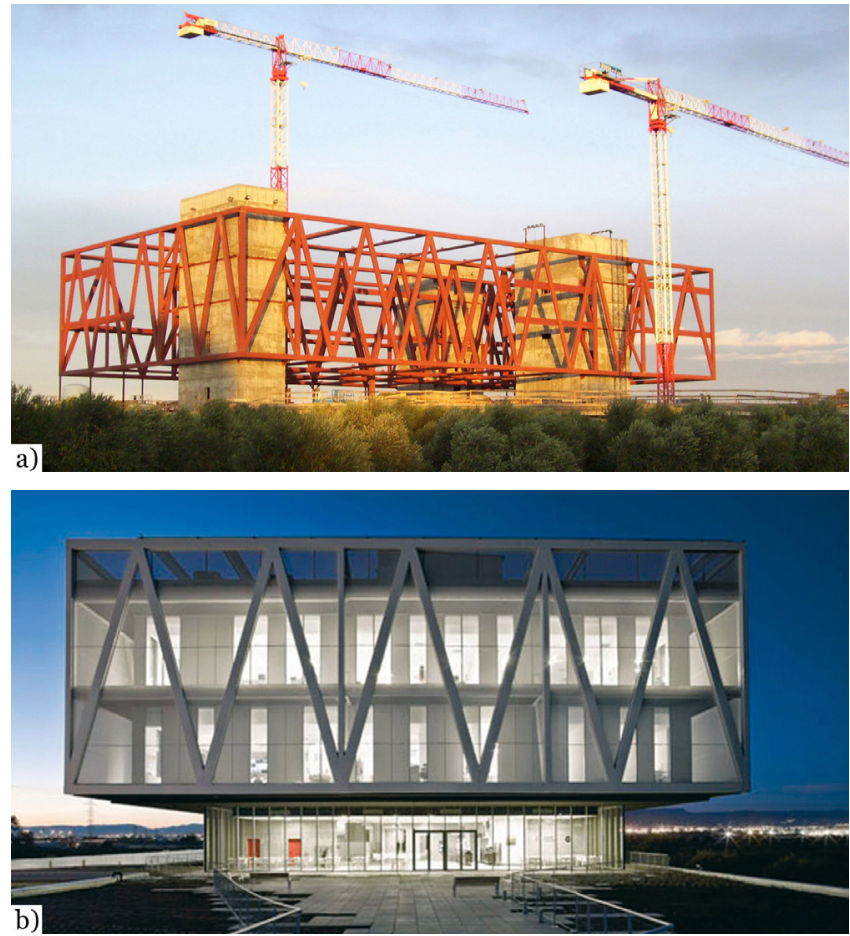

Figura 8. a) Vista general de la estructura metálica en la fase final de su ejecución, b) Vista de la fachada lateral este del edificio 112 Reus. (Imágenes propia y cedida por Adrià Goula, respectivamente).

\section{AGRADECIMIENTOS}

En primer lugar agradecer a la empresa IDOM la disposición de los medios humanos y técnicos requeridos en mi etapa como consultor de estructuras arquitectónicas en ACXTIDOM. Algunos de los resultados de este prolífero período se muestran en las realizaciones propias (figuras), fruto del proceso natural de desarrollo y definición de una estructura resistente de estas características.

En este sentido, hacer una mención especial a los arquitectos Marco Suárez y Roberto Molinos por compartir tantas reflexiones sobre conceptos arquitectónicos y estructurales avanzados, surgidas de las largas, y tendidas, conversaciones mantenidas. Asimismo, agradecer al resto de profesionales que participaron en el proyecto por su disposición a trabajar en equipo en todo momento.

Por último, agradecer al arquitecto Adrià Goula su aportación con las fotos cedidas del edificio 112 Reus, que han permitido documentar gráficamente parte del presente artículo.

\section{REFERENCIAS}

(1) Suárez, M. (2011). Centro de Atención y Gestión de Llamadas de Urgencia 112 Catalunya. Edificio 112 Reus. Convocatoria para la selección de edificios españoles para participar en SB11 Helsinki. Finlandia, Helsinki.

(2) Sans, S. (2009, 19 de octubre). Más de 50 millones para el edificio 112 de Reus. LaVanguardia.es.

(3) Basset, L., Guardiola, A. (2011). Celosías. Valencia: Universitat Politècnica de València (RiuNet repositorio).

(4) Señís, R., Sastre, R., Brufau, R., Carbajal, E.C. (2014). Estudio para la optimización de mallas estructurales de acero envolventes de edificios en altura según sus solicitaciones, en base al análisis de sus líneas isostáticas. Informes de la Construcción, 66(extra-1): mo05, doi: http://dx.doi.org/10.3989/ic.13.094.

(5) Señís, R. (2014). Utilització de les línies isostàtiques com a criteri de disseny per a millorar l'eficiència de la trajectòria de les malles estructurals d'acer envolupants d'edificis en alçada. Quaderns d'Estructures, (51): 38-44.

(6) Señís, R. (2013). Isostatic lines' study to optimize steel space grid envelope structures for tall buildings according to their solicitations. En Proceedings of the 2nd International Conference on Structures and Architecture, (pp. 631-635). Guimarães: CRC. 
(7) Señís, R., Sastre, R., Brufau, R., Carbajal, E.C. (2014). Ensayos con túnel de viento de edificios "romo" de base circular para optimizar mallas espaciales envolventes de edificios en altura. En Actas del VI Congreso Internacional de Estructuras de ACHE, (pp. 1-6). Madrid.

(8) Señís, R. (2014). Optimización de mallas estructurales de acero envolventes de edificios en altura. Análisis de las direcciones principales identificadas por sus líneas isostáticas (Tesis doctoral). Barcelona: Universitat Politècnica de Catalunya. Id: http://www.tdx.cat/handle/10803/146178.

(9) Aguiló, M., Manterola, J., Onzain, M., Rui-Wamba, J. (2004). Javier Manterola Armisén. Pensamiento y obra. Madrid: Fundación Esteyco.

(10) Manterola, J. (2005). La estructura resistente en la arquitectura actual (continuación). Informes de la Construcción, 57(499-500): 9-35, doi: http://dx.doi.org/10.3989/ic.2005.v57.i499-500.480.

(11) Bernabeu, A. (2007). Estrategias de diseño estructural en la arquitectura contemporánea. El trabajo de Cecil Balmond (Tesis doctoral). Madrid: Universidad Politécnica de Madrid.

(12) Aguiló, M. (2002). Invariantes estéticos de la Ingeniería Civil. Revista de Obras Públicas, (3426): 59-68.

(13) Brufau, R. (2004). La flexió a les estructures metàl·liques. Les encavallades. Barcelona: Escola Professional Josep Ma Sert, COAC.

(14) Estévez, J., Martín, E. (2002). Influencia de los parámetros de diseño en el rendimiento estructural de vigas de celosía. Informes de la Construcción, 54(479): 17-28, doi: http://dx.doi.org/10.3989/ic.2002.v54.i479.617.

(15) Goñi, R. (2007). Cómo construir un edificio-puente. Aspectos estructurales. Revista de Edificación, (36-37): 31-36.

(16) Muttoni, A. (2011). The Art of Structures. Introduction to the Functioning of Structures in Architecture. Laussane: EPFL Press.

(17) Señís, R., Brufau, R., Sastre, R., Carbajal, E.C. (2015). Comparative study of trusses to determine the influence of geometry in the structural efficiency, according to the directions of the principal stresses. En Proceedings of the 3rd International Conference on Mechanical Models in Structural Engineering, (pp. 159-168). Sevilla.

(18) Estévez, J., Martín, E. (2003). Análisis del rendimiento estructural de vigas Vierendeel. Informes de la Construcción, 54(483): 27-37, doi: http://dx.doi.org/10.3989/ic.2003.v54.i483.573.

(19) Escrig, F., Sánchez, J. (2004). Diseño y análisis de una gran cubierta y criterios de diseño. Informes de la Construcción, 55(490): 29-37, doi: http://dx.doi.org/10.3989/ic.2004.v55.i490.418.

(20) de Jager, B., Skelton R.E. (2006). Stiffness of planar tensegrity truss topologies. International Journal of Solids and Structures, 43(5): 1308-1330, doi: http://dx.doi.org/10.1016/j.ijsolstr.2005.06.049.

(21) Xu, W., Han, L.-H., Tao, Z. (2014). Flexural behaviour of curved concrete filled steel tubular trusses. Journal of Constructional Steel Research, 93: 119-134, doi: http://dx.doi.org/10.1016/j.jcsr.2013.10.015.

(22) Mazurek, A., Baker, W.F., Tort, C. (2011). Geometrical aspect of optimum truss like structures. Structural and Multidisciplinary optimization, 43(2): 231-242, doi: http://dx.doi.org/10.1007/s00158-010-0559-x.

(23) Fenton, M., McNally, C., Byrne, J., Hemberg, E., McDermott, J., O’Neill, M.(2014). Automaticinnovative truss design using grammatical evolution. Automation in Construction, 39: 59-69, doi: http://dx.doi.org/10.1016/j.autcon.2013.11.009.

(24) Beghini, L.L., Carrion, J., Beghini, A., Mazurek, A., Baker, W.F. (2014). Structural optimization using graphic statics. Structural and Multidisciplinary optimization, 49(3): 351-366, doi: http://dx.doi.org/10.1007/s00158-013-1002-x.

(25) Descamps, B., Coelho, R.F. (2014). The nominal force method for truss geometry and topology optimization incorporating stability considerations. International Journal of Solids and Structures, 51(13): 2390-2399, doi: http://dx.doi. org/10.1016/j.ijsolstr.2014.03.003.

(26) Ministerio de Fomento - CTE. (2006). Documento Básico SE-AE (Seguridad Estructural: Bases de Cálculo y Acciones en la edificación). Madrid: Código Técnico de la Edificación.

(27) Ministerio de Fomento. (2002). Norma de Construcción Sismorresistente: Parte General y Edificación (NCSE-o2). Madrid: Centro de Publicaciones Secretaría General Técnica - Ministerio de Fomento.

(28) de Miguel, A., de la Fuente, P. (2011). Análisis dinámico de estructuras en el dominio de la frecuencia. En Proceedings of the 1st International Congress on Mechanical Models in Structural Engineering, (pp. 15-26). Granada.

(29) AENOR. (1998). UNE-ENV 1998-1-1: Eurocódigo 8. Disposiciones para el proyecto de estructuras sismorresistentes. Parte 1-1: Reglas generales. Acciones sísmicas y requisitos generales de las estructuras. Madrid: Asociación Española de Normalización (AENOR).

(30) Ibrahim, M. (2006). To BIM or not to BIM, this is not the question: how to implement BIM solutions in large design firm environments. En Proceedings of the eCAADe Conference. Volos, Greece. 